\title{
Rogers semilattices of families of two embedded sets in the Ershov hierarchy
}

\author{
Serikzhan A. Badaev*, Mustafa Manat ${ }^{\dagger}$ and Andrea Sorbi ${ }^{\ddagger}$
}

\begin{abstract}
Let $a$ be a Kleene's ordinal notation of a nonzero computable ordinal. We give a sufficient condition on $a$, so that for every $\Sigma_{a}^{-1}$-computable family of two embedded sets, i.e. two sets $A, B$, with $A$ properly contained in $B$, the Rogers semilattice of the family is infinite. This condition is satisfied by every notation of $\omega$; moreover every nonzero computable ordinal that is not sum of any two smaller ordinals has a notation that satisfies this condition. On the other hand, we also give a sufficient condition on $a$, that yields that there is a $\Sigma_{a}^{-1}$-computable family of two embedded sets, whose Rogers semilattice consists of exactly one element; this condition is satisfied by all notations of every successor ordinal bigger than 1, and by all notations of the ordinal $\omega+\omega$; moreover every computable ordinal that is sum of two smaller ordinals has a notation that satisfies this condition. We also show that for every nonzero $n \in \omega$, or $n=\omega$, and every notation of a nonzero ordinal there exists a $\Sigma_{a}^{-1}$-computable family of cardinality $n$, whose Rogers semilattice consists of exactly one element.
\end{abstract}

\section{Introduction}

What is the possible cardinality of the Rogers semilattice of a family of sets in the Ershov hierachy? In this paper we first address the case of families consisting of two embedded sets under inclusion: we give a sufficient condition for an ordinal notation $a$ of a nonzero ordinal, that implies that the Rogers semilattice

*Al-Farabi Kazakh National University, Al-Farabi ave., 71, Almaty, 050038, Kazakhstan. Part of the research contained in this paper was carried out while the first author was GNSAGA-INDAM Visiting Professor at the Department of Mathematics and Computer Science "Roberto Magari" of the University of Siena, Italy, July 2011. The first author wishes to thank INDAM-GNSAGA for supporting the visiting professorship.

†Al-Farabi Kazakh National University, Al-Farabi ave., 71, Almaty, 050038, Kazakhstan. Part of the research contained in this paper was carried out while the second author was visiting the Department of Mathematics and Computer Science "Roberto Magari" of the University of Siena, Italy. The second author wishes to thank the Al-Farabi University for supporting the visit,and the Department of Mathematics and Computer Science "Roberto Magari" of siena for its hospitality.

¥Dipartimento di Scienze Matematiche ed Informatiche "Roberto Magari", Università di Siena, 53100 Siena, Italy. 
of any $\Sigma_{a}^{-1}$-computable family $\mathcal{A}=\{A, B\}$ of two embedded sets (i.e. $A \subset B$, meaning that $A \subseteq B$, and $A \neq B$ ) is infinite. (In fact it embeds the many-one degrees of the c.e. sets: this extends a classical result holding for computably enumerable, or c.e., sets.); or, otherwise, we point out a sufficient condition on $a$ implying that there exists a $\Sigma_{a}^{-1}$-computable family of two embedded sets, whose Rogers semilattice consists of exactly one element. (This generalizes a result of Badaev and Talasbaeva [4], stating that there is a family of $d$-c.e. sets such that the Rogers semilattice of $\mathcal{A}$ consists of exactly one element). On the other hand, there is no problem when we consider families without any structural restrictions: we can easily construct a family consisting of any given number of elements, whose Rogers semilattice has only one element. And we can consider lots of families of c.e. sets with infinite classical Rogers semilattices, thus providing examples of infinite Rogers semilattices for any level of the Ershov hierarchy.

For unexplained notions and results on the theory of numberings, the reader is referred to [8]. In a nutshell, Goncharov and Sorbi's proposal, [9], for generalizing the theory of numberings to different notions of computability consists in the following. Let $\mathcal{C}$ be an abstract "notion" of computability, i.e. a countable class of sets of numbers, and let $\mathcal{A} \subseteq \mathcal{C}$ : then a numbering $\pi: \omega \rightarrow \mathcal{A}$ is $\mathcal{C}$-computable, if $\{\langle k, x\rangle: x \in \pi(k)\} \in \mathcal{C}$. On numberings $\alpha, \beta$ of a family $\mathcal{A}$, one defines $\alpha \leq \beta$ if there is a computable function $f$ such that $\alpha=\beta \circ f$; and $\alpha \equiv \beta$ if $\alpha \leq \beta$ and $\beta \leq \alpha$; for $\mathcal{A} \subseteq \mathcal{C}$, we denote by $\operatorname{Comp}_{\mathcal{C}}(\mathcal{A})$ the set of $\mathcal{C}$-computable numberings of $\mathcal{A}$; we say that $\mathcal{A}$ is $\mathcal{C}$-computable if $\operatorname{Comp}_{\mathcal{C}}(\mathcal{A}) \neq \emptyset$; finally we denote by $\mathfrak{R}_{\mathcal{C}}(\mathcal{A})$ the set of Rogers degrees of the elements of $\operatorname{Comp}_{\mathcal{C}}(\mathcal{A})$, i.e. the set $\operatorname{Comp}_{\mathcal{C}}(\mathcal{A}) / \equiv$; it can be shown that $\mathfrak{R}_{\mathcal{C}}(\mathcal{A})$, if nonempty, is an upper semilattice.

The motivation for the above quoted result by Badaev and Talasbaeva, [4], lies in the fact that in the classical case, i.e. in the case $\mathcal{C}=\Sigma_{1}^{0}$-sets, it is well known ([8]) that the Rogers semilattice of any family $\{A, B\}$, with $A \subset B$, is infinite. Indeed, let $\mathcal{A}=\{A, B\}$, with $A \subset B$ : for every c.e. set $U$, one can define the numbering

$$
\alpha_{U}(k)= \begin{cases}A, & \text { if } k \notin U, \\ B, & \text { if } k \in U,\end{cases}
$$

which is computable, since

$$
\left\{\langle k, x\rangle: x \in \alpha_{U}(k)\right\}=\{\langle k, x\rangle: x \in A \text { or }[x \in B \text { and } k \in U]\}
$$

and the latter set is $\Sigma_{1}^{0}$. It is then easy to see that, for all pairs of c.e. sets $U, V$,

$$
U \leq_{m} V \Leftrightarrow \alpha_{U} \leq \alpha_{V}
$$

(where $\leq_{m}$ denotes many-one reducibility): this provides in fact an upper semilattice embedding of the c.e. $m$-degrees into the Rogers semilattice of the family $\{A, B\}$, showing that this Rogers semilattice is infinite. For details see [8].

This result holds of all abstract notions of computability (in the sense of [9]) with reasonable "closure" properties. Clearly the argument remains valid for 
every notion of computability $\mathcal{C}$ for which, given any c.e. set $U$, the right-hand side of (1.2) is still in $\mathcal{C}$. In particular:

Theorem 1.1. If $\mathcal{C} \in\left\{\Sigma_{n}^{0}, \Pi_{n}^{0}, \Sigma_{n}^{1}, \Pi_{n}^{1}, \Delta_{n}^{1}: n \geq 1\right\}$ then the Rogers semilattice of any $\mathcal{C}$-computable family $\{A, B\}$, with $A \subset B$, is infinite.

Proof. The proof is immediate. Notice that in most cases one can embed upper semilattices of $m$-degrees, that are "bigger" than the upper semilattice of c.e. $m$-degrees: for instance, let $\mathcal{C}=\Sigma_{n}^{0}, n \geq 1$ : if $A, B, U \in \Sigma_{n}^{0}$, then the right-hand side of (1.2) is still in $\Sigma_{n}^{0}$, and thus the upper semilattice of the $\Sigma_{n}^{0} m$-degrees is embeddable into the Rogers semilattice of $\{A, B\}$.

In this paper, we extend Badaev and Talasbaeva's result on families of two embedded sets to all notations of successor ordinals, and to all notations of $\omega+\omega$. The proof is a straightforward generalization of [4]. We show however that this is not true for other limit levels of the Ershov hierarchy, in particular it is not true for the notations of $\omega$.

Finally, if we drop the requirement that the family contain embedded sets, then we can show that at every level of the Ershov hierarchy there are (even infinite) families whose Rogers semilattices are singletons. More precisely, for every nonzero $n \in \omega \cup\{\omega\}$, and for every ordinal notation $a$, with $|a|_{0} \geq 1$, there exists a $\Sigma_{a}^{-1}$-computable family $\mathcal{A}$ of exactly $n$ sets, whose Rogers semilattice $\mathfrak{R}_{a}^{-1}(\mathcal{A})$ consists of one element.

We now briefly review the basic notions concerning ordinal notations, and the Ershov hierarchy. We refer to Kleene's system $O$ of ordinal notations for computable ordinals: for details, see [12]. In particular, for $a \in O$, the symbol $|a|_{O}$ represents the ordinal of which $a$ is a notation; the symbol $<_{O}$ denotes Kleene's partial ordering relation on $O$; moreover, the symbol $t_{0}$ denotes a partial computable function, defined on $O$, such that $\left|a+_{O} b\right|_{O}=|a|_{O}+|b|_{O}$, and $a \leq_{O} a+_{O} b$. We now briefly recall the definition of the Ershov hierarchy, introduced in $[5,6,7]$. Our presentation is based on [10].

Definition 1.2. If $a$ is a notation for a computable ordinal, then a set of numbers $A$ is said to be $\Sigma_{a}^{-1}$ if there are a computable function $f(z, t)$ and a partial computable function $\gamma(z, t)$ such that, for all $z$,

1. $A(z)=\lim _{t} f(z, t)$, with $f(z, 0)=0$; (here, given a set $X$, and a number $z$, the symbol $X(z)$ denotes the value of the characteristic function of $X$ on $z$ );

2. (a) $\gamma(z, t) \downarrow \Rightarrow \gamma(z, t+1) \downarrow \& \gamma(z, t+1) \leq_{O} \gamma(z, t)<_{O} a$;

(b) $f(z, t+1) \neq f(z, t) \Rightarrow \gamma(z, t+1) \downarrow \neq \gamma(z, t)$.

We call the partial function $\gamma$ the mind-change function for A, relatively to $f$.

A $\Sigma_{a}^{-1}$-approximation to a $\Sigma_{a}^{-1}$-set $A$, is a pair $\langle f, \gamma\rangle$, where $f$ and $\gamma$ are respectively a computable function and a partial computable function satisfying 1. and 2., above, for $A$.

Following [9], we give the following: 
Definition 1.3. A $\Sigma_{a}^{-1}$-computable numbering of a family $\mathcal{A}$ of $\Sigma_{a}^{-1}$-sets is an onto function $\pi: \omega \longrightarrow \mathcal{A}$, such that

$$
\{\langle k, x\rangle: x \in \pi(k)\} \in \Sigma_{a}^{-1} .
$$

Hence there exist a computable function $f(z, t)$ and a partial computable function $\gamma(z, t)$, such that $\pi(k)(x)=\lim _{t} f(\langle k, x\rangle, t)$, with $f(z, 0)=0$ for all $z$; and $\gamma$ is the mind-change function for $\{\langle k, x\rangle: x \in \pi(k)\}$ relatively to $f$.

In the rest of the paper we will write $\operatorname{Comp}_{a}^{-1}(\mathcal{A})$ for $\operatorname{Comp}_{\Sigma_{\alpha}^{-1}}(\mathcal{A})$, and $\mathfrak{R}_{a}^{-1}(\mathcal{A})$ for $\mathfrak{R}_{\Sigma_{a}^{-1}}(\mathcal{A})$.

We recall (see e.g. [6]) that there is an indexing $\left\{A_{z}\right\}_{z \in \omega}$ of the family of all $\Sigma_{a}^{-1}$ sets, such that $\left\{\langle x, z\rangle: x \in A_{z}\right\} \in \Sigma_{a}^{-1}$. From this, it is possible (for more details, see [10]) to define an indexing $\left\{\pi_{e}\right\}_{e \in \omega}$ of all computable numberings of families of $\Sigma_{a}^{-1}$ sets, for which

$$
\left\{\langle e, k, x\rangle: x \in \pi_{e}(k)\right\} \in \Sigma_{a}^{-1},
$$

i.e. the set $\left\{\langle e, k, x\rangle: x \in \pi_{e}(k)\right\}$ has a $\Sigma_{a}^{-1}$-approximation $\langle f, \gamma\rangle$ : an indexing satisfying this property is called a $\Sigma_{a}^{-1}$-computable indexing of all $\Sigma_{a}^{-1}$ computable numberings. Clearly, from $e, k$ one has an effective way of getting a $\Sigma_{a}^{-1}$-approximation $\left\langle f_{\pi_{e}(k)}, \gamma_{\pi_{e}(k)}\right\rangle$ to the set $\pi_{e}(k)$.

\section{The theorems}

We begin by showing that there exist (limit) ordinal notations $a$, such that the Rogers semilattice of every $\Sigma_{a}^{-1}$-computable family of two embedded sets is infinite:

Theorem 2.1. If $\mathcal{A}=\{A, B\}$, with $A \subset B$, is a $\Sigma_{a}^{-1}$-computable family, where $|a|_{O}$ is a (necessarily limit) ordinal, and there exists a partial computable function $\psi$ such that for every $b_{0}, b_{1}<_{O}$ a we have that

$$
\psi\left(b_{0}, b_{1}\right) \downarrow<_{O} a \&\left|b_{0}\right|_{O}+_{O}\left|b_{1}\right|_{O}=\left|\psi\left(b_{0}, b_{1}\right)\right|_{O},
$$

then $\mathfrak{R}_{a}^{-1}(\mathcal{A})$ is infinite, in fact it embeds the upper semilattice of the c.e. $m-$ degrees.

Proof. Let $a$ be any notation satisfying the assumptions of the theorem, and let $\mathcal{A}=\{A, B\}$ be a $\Sigma_{a}^{-1}$-computable family, with $A \subset B$. Assume that $\left\langle f_{A}, \gamma_{A}\right\rangle$ and $\left\langle f_{B}, \gamma_{B}\right\rangle$ are $\Sigma_{a}^{-1}$-approximations to $A$ and $B$, respectively.

We need only to show that for every c.e. set $U$, the numbering $\alpha_{U}$, as defined in (1.1) is $\Sigma_{a}^{-1}$-computable. For this, we define a $\Sigma_{a}^{-1}$-approximation $\langle f, \gamma\rangle$ to $\alpha_{U}$ : for simplicity, write $f(k, x, s)$ and $\gamma(k, x, s)$, instead of $f(\langle k, x\rangle, s)$ and $\gamma(\langle k, x\rangle, s)$, respectively. Let assume that $\left\{U_{s}: s \in \omega\right\}$ is a computable approximation to $U$. The idea is to have $\langle f, \gamma\rangle$ resemble $\left\langle f_{A}, \gamma_{A}\right\rangle$ until we do not see $k \in U$, and then have $\langle f, \gamma\rangle$ to switch to resembling $\left\langle f_{B}, \gamma_{B}\right\rangle$. 
At stage $s$ a pair $\langle k, x\rangle$ may be defined $A$-related (meaning intuitively that, in defining $f$ and $\gamma$, the current purpose is to resemble $\left.\left\langle f_{A}, \gamma_{A}\right\rangle\right)$, or $B$-related (meaning that we have decided to switch to $\left\langle f_{B}, \gamma_{B}\right\rangle$ ): precise definitions will be given in the construction.

The construction The construction is by stages: at stage $s$ we define $f(k, x, s)$ and $\gamma(k, x, s)$ for every $k, x$. In the rest of the proof, we write, for $b_{0}, b_{1}<_{O} a$,

$$
b_{0}+{ }_{a} b_{1}=\psi\left(b_{0}, b_{1}\right)
$$

Stage 0. Let $f(k, x, 0)=0$ and $\gamma(k, x, 0) \uparrow$ for every pair $k, x$. No pair at stage 0 is either $A$-related or $B$-related.

Stage $s+1$. We distinguish the two cases, whether or not $k \in U_{s+1}$ :

Case 1: $\quad k \notin U_{s+1}$. Consider in the given order the following subcases:

Subcase 1.1: $\langle k, x\rangle$ is not $A$-related, $\gamma(k, x, s) \uparrow$, and $f_{A}(x, s+1)=f_{B}(x, s+$ 1) $=1$.

In this subcase, $\langle k, x\rangle$ starts to be $A$-related, and we define

$$
\begin{aligned}
& f(k, x, s+1)=1 \\
& \gamma(k, x, s+1)=\gamma_{B}(x, s+1)+{ }_{a} \gamma_{A}(x, s+1)+o 2
\end{aligned}
$$

(Notice that under the assumptions, $\gamma(k, x, s+1)<_{O} a$, since $|a|_{O}$ is limit.)

Subcase 1.2: $\langle k, x\rangle$ is $A$-related. In this subcase, define

$$
\begin{aligned}
& f(k, x, s+1)=f_{A}(k, x, s+1) \\
& \gamma(k, x, s+1)=\gamma_{B}\left(x, t_{0}\right)+{ }_{a} \gamma_{A}(x, s+1)+o 2
\end{aligned}
$$

where $t_{0}$ is the stage at which we have defined $\gamma(k, x, t)$ for the first time.

Subcase 1.3: None of the previous subcases holds. Do nothing, and let

$$
\begin{aligned}
& f(k, x, s+1)=f(k, x, s) \\
& \gamma(k, x, s+1)=\gamma(k, x, s) .
\end{aligned}
$$


Case 2: $k \in U_{s+1}$. Consider the following subcases:

Subcase 2.1: $\langle k, x\rangle$ is $A$-related.

In this subcase, $\langle k, x\rangle$ ceases to be $A$-related and starts to be $B$-related, and we define

$$
\begin{aligned}
& f(k, x, s+1)=f_{B}(x, s+1) \\
& \gamma(k, x, s+1)=\gamma_{B}\left(x, t_{0}\right) .
\end{aligned}
$$

(Notice that this may entail that $f(k, x, s+1)=f(k, x, s)$, but $\gamma(k, x, s+1)<_{0}$ $\gamma(k, x, s)$, which is not ruled out by the definition of a $\Sigma_{a}^{-1}$-approximation.)

Subcase 2.2: $\langle k, x\rangle$ is neither $A$-related nor $B$-related, and $\gamma_{B}(x, s+1) \downarrow$. In this subcase, $\langle k, x\rangle$ starts to become $B$-related, and we define

$$
\begin{aligned}
& f(k, x, s+1)=f_{B}(x, s+1) \\
& \gamma(k, x, s+1)=\gamma_{B}(x, s+1) .
\end{aligned}
$$

Subcase 2.3: $\langle k, x\rangle$ is $B$-related. In this subcase define

$$
\begin{aligned}
& f(k, x, s+1)=f_{B}(x, s+1) \\
& \gamma(k, x, s+1)=\gamma_{B}(x, s+1) .
\end{aligned}
$$

Subcase 2.4: None of the previous subcases holds. Do nothing, and let

$$
\begin{aligned}
& f(k, x, s+1)=f(k, x, s) \\
& \gamma(k, x, s+1)=\gamma(k, x, s) .
\end{aligned}
$$

Verification. We recall the following rule of ordinal arithmetic (see for instance [11]):

$$
\beta<\gamma \Leftrightarrow \alpha+\beta<\alpha+\gamma .
$$

Given $U$ and $k$, it is the clear from the definition that the pair of functions $\langle f, \gamma\rangle$ is a $\Sigma_{a}^{-1}$-approximation to $\alpha_{U}$. Indeed, if $\gamma(k, x, s)$ is always undefined, then for every $s, f(k, x, s)=0$. Otherwise, if $t_{0}$ is the first stage at which $\gamma\left(k, x, t_{0}\right)$ is defined, then $\gamma\left(k, x, t_{0}\right)<_{O} a$. Moreover, while $\langle k, x\rangle$ is $A$-related, if $f(k, x, s+1) \neq f(k, x, s)$, this is due to the fact that $f_{A}(x, s+1) \neq f_{A}(x, s)$, thus $\gamma_{A}(x, s+1)<_{O} \gamma_{A}(x, s)$, which yields by (2.1) that $\gamma(k, x, s+1)<_{O} \gamma(k, x, s)$. A similar argument applies while $\langle k, x\rangle$ is $B$-related. We add $+_{o} 2$ the first time we define $\gamma\left(k, x, t_{0}\right)$ because we need one extra change when passing from $A$-relatedness to $B$-relatedness, which not necessarily corresponds to a change in $\gamma_{B}$.

It remains to show that

$$
\alpha_{U}(k)= \begin{cases}A, & \text { if } k \notin U \\ B, & \text { if } k \in U .\end{cases}
$$

Consider any $k$. We distinguish the following two cases: 
1. $k \notin U$. For any given $x$, if $\langle k, x\rangle$ never becomes $A$-related, then $x \notin A$, since otherwise $x \in B$ too, and at some stage the pair $\langle k, x\rangle$ would become $A$-related. If, on the other hand, $\langle k, x\rangle$ first becomes $A$-related at stage $t_{0}$, then at all stages $s \geq t_{0}, f(k, x, s)=f_{A}(x, s)$. It follows that $\alpha_{U}(k)=A$.

2. If $k \in U$, then either $\langle k, x\rangle$ never becomes $B$-related, which implies that $x \notin B$, and on the other hand $f(k, x, s)=0$ for every $s$, i.e. $x \notin \alpha_{U}(k)$. Or, at some least stage $t_{1}$ (with $t_{1}>t_{0}$, if there has been a least $t_{0}$ at which $\langle k, x\rangle$ became $A$-related) the pair $\langle k, x\rangle$ becomes $B$-related: at all stages $s \geq t_{1}$ we have that $f(k, x, s)=f_{B}(x, s)$. We conclude that $\alpha_{U}(k)=B$.

Remark 2.2. The condition expressed in Theorem 2.1 is satisfied by the ordinal 1. So the previous theorem extends the classical result holding for c.e. sets, namely if $\mathcal{A}$ consists of two embedded c.e. sets, then $\Re_{1}^{-1}(\mathcal{A})$ embeds the c.e. $m$-degrees. $\left(\mathfrak{R}_{1}^{-1}(\mathcal{A})\right.$ is also called $\mathfrak{R}_{1}^{0}(\mathcal{A})$ in $[9]$, or $\mathfrak{R}(\mathcal{A})$ in [8].)

Corollary 2.3. Let a be any notation of $\omega$. Then for every $\Sigma_{a}^{-1}$-computable family $\mathcal{A}=\{A, B\}$, with $A \subset B$ we have that $\mathfrak{R}_{a}^{-1}(\mathcal{A})$ is infinite.

Proof. The proof is obvious: take $\psi=+_{o}$ in this case.

Remark 2.4. We do not know if the conclusion of Theorem 2.1 holds of all notations of any computable ordinal $\alpha$ which is closed under sum. (We say that an ordinal $\alpha$ which is closed under sum if for every $\beta_{0}, \beta_{1}<\alpha$, one has that $\beta_{0}+\beta_{1}<\alpha$.) In particular, do the conclusions of the previous theorem hold for every notation of $\omega^{2}$ ? Pavel Alaev [1] has recently shown that for every computable ordinal which is closed under sum, i.e. computable ordinals of the form $\omega^{\alpha}$ (since, by the Cantor Normal Form, every ordinal can be uniquely written as $\omega^{\beta_{1}} n_{1}+\cdots+\omega^{\beta_{k}} n_{k}$, with $\beta_{1}>\cdots>\beta_{k}$, and each $\left.n_{i} \in \omega\right)$ there exists (uniformly in a notation for $\alpha$ ) a notation $a$ and a partial computable function $\psi$ as in the statement of Theorem 2.1.

As an example we show here that there is such a notation $a$ for $\omega^{2}$. Let $i_{0}$ be an index of the computable function such that, for every $n, \varphi_{i_{0}}(n)$ is a notation of the ordinal $n$. By the $s-m-n$-theorem, let $s$ be a 1-1 computable function such that

$$
\varphi_{s(i)}(n)= \begin{cases}3 \cdot 5^{i}, & \text { if } n=0 \\ 2^{\varphi_{i}(u)}, & \text { if } n=u+1\end{cases}
$$

Then

$$
\begin{aligned}
1 & <_{O} 2<_{O} 2^{2}<_{O} \cdots \\
& <_{O} 3 \cdot 5^{i_{0}}<_{O} 2^{3 \cdot 5^{i_{0}}}<_{O} 2^{2^{3 \cdot 5^{i} 0}}<_{O} \cdots \\
& <_{O} 3 \cdot 5^{s\left(i_{0}\right)}<_{O} 2^{3 \cdot 5^{s\left(i_{0}\right)}}<_{O} 2^{2^{3 \cdot 5^{s\left(i_{0}\right)}}}<_{O} \cdots \\
& <_{O} 3 \cdot 5^{s^{2}\left(i_{0}\right)}<_{O} 2^{3 \cdot 5^{s^{2}\left(i_{0}\right)}}<_{O} 2^{2^{3 \cdot 5^{s^{2}\left(i_{0}\right)}}}<_{O} \cdots \\
& <_{O} \cdots
\end{aligned}
$$


are notations that list the ordinals below $\omega^{2}$. Let $a=3 \cdot 5^{y}$, where $\varphi_{y}(n)=$ $3 \cdot 5^{s^{n}\left(i_{0}\right)}$ : hence $a$ is a notation for $\omega^{2}$. At this point it is easy to show that there exists a suitable partial computable function $\psi$, as requested by the statement of Theorem 2.1: indeed, given $b_{0}, b_{1}<_{O} a$, it is possible to effectively locate a notation for their ordinal sum in the above list.

Corollary 2.5. For every nonzero computable ordinal $\alpha$ that is closed under sum, there exists a notation a for $\alpha$ such that the Rogers semilattice of any $\Sigma_{a}^{-1}$-computable family of two embedded sets, is infinite.

Proof. By Theorem 2.1 and Remark 2.4.

Next theorem provides sufficient conditions that imply that there exists a $\Sigma_{a}^{-1}$-computable family $\mathcal{A}=\{A, B\}$, with $A \subset B$, whose Rogers semilattice has cardinality 1.

Theorem 2.6. Suppose that a is an ordinal notation such that there exist notations $b_{0}, b_{1}$ with:

1. $b_{0}, b_{1}<_{O} a$ and $b_{0}+_{O} b_{1} \geq_{O} a$;

2. there exists a partial computable function $\psi$ such that, for every $c$, if $b_{0} \leq_{O}$ $c<_{O}$ a then $\psi(c) \downarrow$ and

$$
b_{0}+o \psi(c)=c .
$$

Then there exists a $\Sigma_{a}^{-1}$-computable family $\mathcal{A}=\{A, B\}$, with $A \subset B$ such that $\left|\Re_{a}^{-1}(\mathcal{A})\right|=1$.

Proof. Given $a \in O$, as in the hypotheses of the theorem, we build $A \subset B$, and a $\Sigma_{a}^{-1}$-computable numbering $\alpha$ of $\mathcal{A}=\{A, B\}$, such that for every $\Sigma_{a}^{-1}$ computable numbering $\pi$ of $\mathcal{A}$, we have $\pi \equiv \alpha$. We will define $\alpha(0)=A$, and $\alpha(k)=B$ for all $k \geq 1$.

Let $\left\{\pi_{e}\right\}_{e \in \omega}$ be a $\Sigma_{a}^{-1}$-computable indexing of all $\Sigma_{a}^{-1}$-computable numberings, with uniform $\Sigma_{a}^{-1}$-approximation given by $\langle f, \gamma\rangle$, from which one uniformly gets a $\Sigma_{a}^{-1}$-approximation $\left\langle f_{\pi_{e}(k)}(x, s), \gamma_{\pi_{e}(k)}(x, s)\right\rangle$ to each set $\pi_{e}(k)$. We will define a numbering $\alpha$ so that, for every $e, k$, the following requirement is satisfied:

$$
Q_{e, k}: \quad \pi_{e}(k) \in\{A, B\} \Rightarrow g_{e}(k) \downarrow \& \pi_{e}(k)=\alpha\left(g_{e}(k)\right),
$$

where $g_{e}$ is a partial computable function defined by us. Thus, if $\pi_{e}$ is a numbering of $\{A, B\}$ then $g_{e}$ is total and $\pi_{e}=\alpha \circ g_{e}$

The construction is by stages. Let

$$
R_{e, k}=\{\langle e, k, t\rangle+1: t \in \omega\}:
$$

at stage $s+1$, with $s \in R_{e, k}$ (notice that for every $t>0$ there is a unique pair $e, k$ such that $t \in R_{e, k}$ ) our action aims first at making $\pi_{e}(k) \notin \mathcal{A}$ (so that $\pi_{e}$ is not a numbering of $\mathcal{A}$ ), or, if otherwise $\pi_{e}(k) \in \mathcal{A}$, we define $g_{e}(k) \in\{0,1\}$ so as to ensure that $\pi_{e}(k)=\alpha\left(g_{e}(k)\right)$. 
Our attempts at diagonalizing $\pi_{e}(k)$ against $A, B$ at stage $s+1$ with $s \in$ $R_{e, k}$, make use of (computably given) witnesses $a_{0}(e, k), a_{1}(e, k)$ : we assume that $a_{0}(e, k) \neq a_{1}(e, k)$, and $\left\{a_{0}(e, k), a_{1}(e, k)\right\} \cap\left\{a_{0}\left(e^{\prime}, k^{\prime}\right), a_{1}\left(e^{\prime}, k^{\prime}\right)\right\}=\emptyset$ if $\langle e, k\rangle \neq\left\langle e^{\prime}, k^{\prime}\right\rangle$. At stage $s$ we define values $f_{A}\left(a_{0}(e, k), s\right), f_{A}\left(a_{1}(e, k), s\right)$, and $\gamma_{A}\left(a_{0}(e, k), s\right), \gamma_{A}\left(a_{1}(e, k), s\right)$; and, similarly, we also define $f_{B}\left(a_{0}(e, k), s\right)$, $f_{B}\left(a_{1}(e, k), s\right)$ and $\gamma_{B}\left(a_{0}(e, k), s\right), \gamma_{B}\left(a_{1}(e, k), s\right)$. The pairs $\left\langle f_{A}, \gamma_{A}\right\rangle$ and $\left\langle f_{B}, \gamma_{B}\right\rangle$ will be $\Sigma_{a}^{-1}$-approximations to $A$ and $B$, respectively. From these two pairs we will also get a $\Sigma_{a}^{-1}$-approximation $\langle\hat{f}, \hat{\gamma}\rangle$ to $\alpha$, by letting

$$
\hat{f}(\langle k, x\rangle, s)= \begin{cases}f_{A}(x, s), & \text { if } k=0 \\ f_{B}(x, s), & \text { if } k \geq 1,\end{cases}
$$

and

$$
\hat{\gamma}(\langle k, x\rangle, s)= \begin{cases}\gamma_{A}(x, s), & \text { if } k=0 \\ \gamma_{B}(x, s), & \text { if } k \geq 1 .\end{cases}
$$

It is understood that all values of $f_{A}, f_{B}, \gamma_{A}, \gamma_{B}$ that are not explicitly defined at a given stage, maintain the same values (including also when they are undefined) as at the preceding stage, the values at $s=0$ being 0 for $f_{A}, f_{B}$, and undefined for $\gamma_{A}, \gamma_{B}$. Finally, at stage $s+1$, with $s \in R_{e, k}$, if $g_{e, s}(k) \uparrow$ (i.e. the value of $g_{e}(k)$ is still undefined), we might define $g_{e, s+1}(k)=0$ or $g_{e, s+1}(k)=1$. After defining $g_{e}(k)$ our only worry will be to make sure that $\pi_{e}(k) \neq A$ if $g_{e}(k)=1$, and $\pi_{e}(k) \neq B$ if $g_{e}(k)=0$.

Without loss of generality we may also assume that our approximation $\langle f, \gamma\rangle$ also satisfies, for every $e, k, x, f_{\pi_{e}(k)}(x, 1)=0$.

So, let $a, b_{0}, b_{1}$ be ordinal notations, and let $\psi$ be a partial computable function, as in the statement of the theorem. We will write $\psi(c)=c-{ }_{a} b_{0}$, whenever $\psi(c)$ is defined.

The construction The construction is by stages.

Stage 0: Let $f_{A}(z, 0)=f_{B}(z, 0)=0$ and let $\gamma_{A}(z, 0)$ and $\gamma_{B}(z, 0)$ be undefined.

Stage 1: for every $e, k$, let

$$
\begin{aligned}
& f_{A}\left(a_{0}(e, k), 1\right)=1 \\
& \gamma_{A}\left(a_{0}(e, k), 1\right)=b_{1}
\end{aligned}
$$

and

$$
\begin{aligned}
& f_{B}\left(a_{0}(e, k), 1\right)=f_{B}\left(a_{1}(e, k), 1\right)=1 \\
& \gamma_{B}\left(a_{0}(e, k), 1\right)=b_{0} \\
& \gamma_{B}\left(a_{1}(e, k), 1\right)=1 .
\end{aligned}
$$


Stage $s+1, s>0$ : Suppose $s \in R_{e, k}$. For simplicity let $a_{i}=a_{i}(e, k)$. Consider the following cases, and act according to the first one that applies:

1. if $g_{e, s}(k) \uparrow$ and $f_{\pi_{e}(k)}\left(a_{1}, s+1\right)=1$ then define $g_{e, s+1}(k)=1$, and let

$$
\begin{aligned}
& f_{A}\left(a_{0}, s+1\right)=0, \\
& \gamma_{A}\left(a_{0}, s+1\right)=1
\end{aligned}
$$

(notice, the construction will guarantee that $\gamma_{A}\left(a_{0}, s\right)>_{O} 1$ if $f_{A}\left(a_{0}, s\right)=$ $1 ;)$

2. if $g_{e, s}(k)=1$ and $f_{\pi_{e}(k)}\left(a_{1}, s+1\right)=f_{A}\left(a_{1}, s\right)$ then define

$$
\begin{aligned}
& f_{A}\left(a_{1}, s+1\right)=1-f_{A}\left(a_{1}, s\right), \\
& \gamma_{A}\left(a_{1}, s+1\right)=\gamma_{\pi_{e}(k)}\left(a_{1}, s+1\right) ;
\end{aligned}
$$

(notice, $f_{\pi_{e}(k)}\left(a_{1}, u\right)$ has changed already at least twice, so $\gamma_{\pi_{e}(k)}\left(a_{1}, s+1\right)$ is defined;)

3. if $g_{e, s}(k) \uparrow, f_{\pi_{e}(k)}\left(a_{0}, s+1\right)=f_{A}\left(a_{0}, s\right)$, and $\gamma_{\pi_{e}(k)}\left(a_{0}, s+1\right)>_{O} b_{0}$, then let

$$
\begin{aligned}
& f_{A}\left(a_{0}, s+1\right)=1-f_{A}\left(a_{0}, s\right) \\
& \gamma_{A}\left(a_{0}, s+1\right)=\gamma_{\pi_{e}(k)}\left(a_{0}, s+1\right)-{ }_{a} b_{0}
\end{aligned}
$$

4. if $g_{e, s}(k) \uparrow$, and $s+1$ is the first stage in $R_{e, k}$ at which $\gamma_{\pi_{e}(k)}\left(a_{0}, s+1\right) \leq_{O}$ $b_{0}$, then let

$$
\begin{aligned}
& f_{A}\left(a_{0}, s+1\right)=0 \\
& \gamma_{A}\left(a_{0}, s+1\right)=1
\end{aligned}
$$

(notice that $\gamma_{A}\left(a_{0}, s\right) \geq_{O} \gamma_{\pi_{e}(k)}\left(a_{0}, s\right)-{ }_{a} b_{0}>_{O} 1$; notice also that it is possible that $f_{A}\left(a_{0}, s\right)=0$, so we change $\gamma_{A}\left(a_{0}, s+1\right)$, even if $f_{A}\left(a_{0}, s+1\right)$ does not change;

5. if $g_{e, s}(k) \uparrow$, case 4 does not apply, and $f_{\pi_{e}(k)}\left(a_{0}, s+1\right)=0$, then let $g_{e, s+1}(k)=0$;

6. if $g_{e, s}(k)=0$, and $f_{\pi_{e}(k)}\left(a_{0}, s+1\right)=f_{B}\left(a_{0}, s\right)$, then let

$$
\begin{aligned}
& f_{B}\left(a_{0}, s+1\right)=1-f_{B}\left(a_{0}, s\right), \\
& \gamma_{B}\left(a_{0}, s+1\right)=\gamma_{\pi_{e}(k)}\left(a_{0}, s+1\right) .
\end{aligned}
$$

(As $f_{B}\left(a_{0}, s\right)=1$, it follows that $f_{\pi_{e}(k)}\left(a_{0}, s+1\right)$ has made at least one more change since when we set $g_{e}\left(a_{0}\right)=0$ : thus $\gamma_{\pi_{e}(k)}\left(a_{0}, s+1\right)<_{O} b_{0}$.) 
Verification. Let $e, k$ be given: we want to show that either $\pi_{e}(k) \notin\{A, B\}$, or $\pi_{e}(k)=\alpha\left(g_{e}(k)\right)$. Again for simplicity, we write $a_{i}=a_{i}(e, k)$. In the rest of the proof, we often drop mentioning the stage (thus writing for instance $g_{e}(k)$ instead of $\left.g_{e, s}(k)\right)$ when this can be done without any danger of confusion.

Notice first that eventually $a_{1} \in B$, since we never change $f_{B}\left(a_{1}\right)$ at any $s \geq 1$ : consistently, at every $s \geq 1$ we have $\gamma_{B}\left(a_{1}\right)=1$. Also, $f_{B}\left(a_{0}\right)$ may change only because of actions taken at item 6 of the construction.

We say that $\pi_{e}(k)$ enters a " $g_{e}(k)=1$ "-setup at stage $s+1$, if at stage $s+1$ we define $g_{e}(k)=1$.

If $\pi_{e}(k)$ enters a " $g_{e}(k)=1$ "-setup at stage $s_{0}+1$, then our actions in the construction guarantee that $g_{e}(k)=1$, and for all $s \geq s_{0}, f_{A}\left(a_{0}, s+1\right)=0$, and $\gamma_{A}\left(a_{0}, s\right)=1$. We change $f_{A}\left(a_{1}\right)$ after defining $g_{e}(k)=1$, only at stages at which we see that some additional change has occurred in $f_{\pi_{e}(k)}\left(a_{1}\right)$ since last time we attacked requirement $Q_{e, k}$, thus $\gamma_{\pi_{e}(k)}\left(a_{1}\right)$ has dropped down; since at any such stage, we redefine $\gamma_{A}\left(a_{1}\right)=\gamma_{\pi_{e}(k)}\left(a_{1}\right)$, it follows that $\gamma_{A}\left(a_{1}\right)$ drops down in the $<_{O}$-ordering consistently with making the pair $\left\langle f_{A}, \gamma_{A}\right\rangle$ a $\Sigma_{a}^{-1}$-approximation. Moreover we also guarantee that $A\left(a_{1}\right) \neq \pi_{e}(k)\left(a_{1}\right)$, and $a_{0} \in B$ (as already remarked, we are allowed to modify $f_{B}\left(a_{0}\right)$ only through item 6 of the construction, which never applies in this case). In conclusion, $\pi_{e}(k) \neq A, A \cap\left\{a_{0}, a_{1}\right\} \subset B \cap\left\{a_{0}, a_{1}\right\}$. Thus if $\pi_{e}(k) \in \mathcal{A}$, then $\pi_{e}(k)=B$, hence $\pi_{e}(k)=\alpha\left(g_{e}(k)\right)$.

Otherwise, suppose that $\pi_{e}(k)$ never enters a " $g_{e}(k)=1$ "-setup. Thus, if there exists a least stage $s_{0}+1$ at which either $a_{0}$ or $a_{1}$ enters $\pi_{e}(k)$, we have that at this stage only $a_{0}$ enters $\pi_{e}(k)$, and at next stage $s+1$ with $s \in R_{e, k}$, the construction responds by making $f_{A}\left(a_{0}\right)=0$ and, if $\gamma_{\pi_{e}(k)}\left(a_{0}\right)>_{O} b_{0}$, by making $\gamma_{A}\left(a_{0}\right)=\gamma_{\pi_{e}(k)}\left(a_{0}\right)-{ }_{a} b_{0}$. Since we assume that $\pi_{e}(k)$ never enters a " $g_{e}(k)=1$ "-setup, at all subsequent stages at which $g_{e}(k)$ is still undefined and $\pi_{e}(k)$ changes on either $a_{0}$ or $a_{1}$, we only have a change on $a_{0}$, at which we respond at next stage $s+1$ with $s \in R_{e, k}$, by diagonalizing $f_{A}\left(a_{0}\right) \neq f_{\pi_{e}(k)}\left(a_{0}\right)$, and decreasing $\gamma_{A}\left(a_{0}\right)$ by defining $\gamma_{A}\left(a_{0}\right)=\gamma_{\pi_{e}(k)}\left(a_{0}\right)-{ }_{a} b_{0}$, as guaranteed by the rule of ordinal arithmetic (2.1), since $\gamma_{\pi_{e}(k)}\left(a_{0}\right)$ has certainly dropped down since last time we attacked requirement $Q_{e, k}$. While in the course of this diagonalization loop, $\pi_{e}(k)$ is neither $A$ nor $B$ (since $a_{1} \notin \pi_{e}(k)$ but $a_{1} \in B$ ). This loop ends at the first subsequent stage $s_{1}+1$ with $s_{1} \in R_{e, k}$ at which $\gamma_{\pi_{e}(k)}\left(a_{0}\right) \leq_{O} b_{0}$ : at this stage we define the final values $g_{e}(k)=0, a_{0} \notin A$, and $\gamma_{A}\left(a_{0}\right)=1$. After this stage, the construction guarantees that $\pi_{e}(k) \neq B$, by diagonalizing $f_{B}\left(a_{0}\right) \neq f_{\pi_{e}(k)}\left(a_{0}\right)$, and decreasing $\gamma_{B}\left(a_{0}\right)$ by defining $\gamma_{B}\left(a_{0}\right)=$ $\gamma_{\pi_{e}(k)}\left(a_{0}\right)$, following, as before, corresponding decreases of $\gamma_{\pi_{e}(k)}\left(a_{0}\right)$ (again, this is guaranteed by the rule of ordinal arithmetic (2.1); our delayed definition of the first value of $\gamma_{B}\left(a_{0}\right)$ guarantees that for the first defined value of $\gamma_{B}\left(a_{0}\right)$ we have $\gamma_{B}\left(a_{0}\right)<_{O} b_{0}$, as explained in the parenthetical remark in item 4). Notice that in this case $A \cap\left\{a_{0}, a_{1}\right\} \subset B \cap\left\{a_{0}, a_{1}\right\}$ since $A \cap\left\{a_{0}, a_{1}\right\}=\emptyset$. Thus, eventually, if $\pi_{e}(k) \in\{A, B\}$ then $\pi_{e}(k)=A$, yielding $\pi_{e}(k)=\alpha\left(g_{e}(k)\right)$ as desired.

Corollary 2.7. Suppose that $\alpha$ is a computable ordinal, such that there exist 
$\beta_{0}, \beta_{1}<\alpha$ with $\alpha=\beta_{0}+\beta_{1}$. Then there exist a notation a of $\alpha$, and a $\Sigma_{a}^{-1}$ computable family $\mathcal{A}=\{A, B\}$, with $A \subset B$ such that $\left|\mathfrak{R}_{a}^{-1}(\mathcal{A})\right|=1$.

Proof. Let $\alpha=\beta_{0}+\beta_{1}$, with $\beta_{0}, \beta_{1}<\alpha$, and let $b_{0}$ be a notation for $\beta_{0}$, and $b_{1}$ a notation for $\beta_{1}$. Then $a=b_{0}+_{o} b_{1}$ is a notation for $\alpha$. A closer look at the definition of $+_{O}$ (see e.g. [3, Proposition 4.18]) shows that for every $c$ such that $b_{0} \leq_{O} c \leq_{O} b_{0}+_{O} b_{1}$ there exists $d \leq_{O} b_{1}$ such that $c=b_{0}+o d$. By properties of $\leq_{O}$, it is clear that given $c$ such that $b_{0} \leq_{O} c \leq_{O} b_{0}+{ }_{O} b_{1}$, one can effectively find $d$ such that $c=b_{0}+o d$, thus there exists a partial computable function $\psi$ such that if $b_{0} \leq_{O} c \leq_{O} b_{0}+_{O} b_{1}$, then $\psi(c) \downarrow$ and $b_{0}+_{O} \psi(c)=c$.

Remark 2.8. Inspection of proofs shows that $B \in \Sigma_{b_{0}+o 2}^{-1}$.

Preliminary to next corollary, we show that we can always assume to work with a $\Sigma_{a}^{-1}$-approximation $\langle f, \gamma\rangle$, in which $\gamma$ is synchronized with $f$, i.e.

$$
\gamma(x, s+1)<_{O} \gamma(x, s) \Leftrightarrow f(x, s+1) \neq f(x, s)
$$

(where $\gamma(x, s+1)<_{O} \gamma(x, s)$ includes also the case of $\gamma(x, s+1)$ defined and $\gamma(x, s)$ undefined).

Lemma 2.9. From any $\Sigma_{a}^{-1}$-approximation $\langle f, \gamma\rangle$ to a set $A$, one can effectively find a $\Sigma_{a}^{-1}$-approximation $\langle f, \hat{\gamma}\rangle$ in which $\hat{\gamma}$ is synchronized with $f$.

Proof. Let $\hat{\gamma}(x, s) \uparrow$, if for all $t \leq s, f(x, t)=0$. If $s_{0}$ is the least stage, if any, at which $f(x, t)=1$, then let $\hat{\gamma}\left(x, s_{0}\right)=\gamma\left(x, s_{0}\right)$. For every $s \geq s_{0}$, let

$$
\hat{\gamma}(x, s+1)= \begin{cases}\hat{\gamma}(x, s), & \text { if } f(x, s+1)=f(x, s), \\ \gamma(x, s+1), & \text { if } f(x, s+1) \neq f(x, s) .\end{cases}
$$

Corollary 2.10. If $a=2^{b_{0}}$ and $b_{0}=2^{c}$ is itself a notation for a successor ordinal, then there exists a $\Sigma_{a}^{-1}$-computable family $\{A, B\}$ of embedded sets, with one-element Rogers semilattice, such that $B \in \Sigma_{b_{0}}^{-1}$. In particular, if $a=2^{2}$, then in accordance with [4] we may construct $B$ as a c.e. set.

Proof. Carry out the same construction as in the proof of the Theorem 2.1 relatively to $a$ and the pair $b_{0}, b_{1}$ where $b_{1}=2$, and originally define $\gamma_{B}\left(a_{0}(e, k), 1\right)=$ $c$; assume that $\gamma$ is synchronized with $f$. Now, let $e, k$ be given, and suppose that at some stage we define $g_{e}(k)=0$ : this is because the first stage at which we have $\gamma_{\pi_{e}(k)}\left(a_{0}(e, k)\right) \leq_{O} b_{0}$, we also have $f_{\pi_{e}(k)}\left(a_{0}(e, k)\right)=1$, by the synchronizing properties of $\gamma_{\pi_{e}(k)}$; at next stage at which we attack requirement $Q_{e, k}$ and we see $f_{\pi_{e}(k)}\left(a_{0}(e, k)\right)=0$, we define $g_{e}(k)=0$; finally, it is only at next stage at which we attack again $Q_{e, k}$ and we see $f_{\pi_{e}(k)}\left(a_{0}(e, k)\right)=1$, that we start to redefine $\gamma_{B}\left(a_{0}(e, k)\right.$, but at this point we have $\gamma_{\pi_{e}(k)}\left(a_{0}(e, k)\right)<_{O} c$, as $f_{\pi_{e}(k)}\left(a_{0}(e, k)\right)$ has changed at least three times. 
Corollary 2.11. If $a$ is a notation of an ordinal $\beta+\omega$, with $\beta, \omega<\beta+\omega$, then there exists a $\Sigma_{a}^{-1}$-computable family $\mathcal{A}=\{A, B\}$, with $A \subset B$ such that $\left|\Re_{a}^{-1}(\mathcal{A})\right|=1$.

Proof. If $a$ is a notation of $\beta+\omega$, with $\omega+1<\beta+\omega$, then let $b_{0}, b_{1}<_{O} a$ be such that $\left|b_{0}\right|_{O}=\beta$ and $\left|b_{1}\right|_{O}=\omega$. To show that a suitable partial computable function $\psi$ as in the statement of Corollary 2.7 exists, take $\psi(c)=u$, if $c=$ $b_{0}+_{O} u$ : use the fact that $\left|b_{0}+_{O} u\right|_{O}=\beta+n$ for some natural number $n$.

Corollary 2.12. If $|a|_{O}=\omega+\omega$, then there exists a $\Sigma_{a}^{-1}$-computable family $\mathcal{A}=\{A, B\}$, with $A \subset B$ such that $\left|\Re_{a}^{-1}(\mathcal{A})\right|=1$.

Proof. Trivial, by the previous corollary.

The following theorem (which follows along the lines of a similar theorem proved by Badaev and Talasbaeva in [4] for all finite levels of the Ershov hierarchy) shows that there is no problem when we consider families without any structural restrictions: we can easily construct a family consisting of any given number of elements whose Rogers semilattice consists of one element.

Theorem 2.13. For every nonzero $n \in \omega \cup\{\omega\}$, and for every ordinal notation a of a nonzero ordinal, there exists a $\Sigma_{a}^{-1}$-computable family $\mathcal{A}$ of exactly $n$ sets, such that $\left|\mathfrak{R}_{a}^{-1}(\mathcal{A})\right|=1$.

Proof. Suppose that we are given a notation $a$ for a computable ordinal $\geq 1$. We begin with building a $\Sigma_{a}^{-1}$-computable Friedberg (i.e. injective) numbering $\alpha$ of an infinite family $\mathcal{A}$ such that, for every $k$, the requirement $R_{k}$,

$$
R_{k}: \quad \pi_{k} \in \operatorname{Comp}_{a}^{-1}(\mathcal{A}) \Rightarrow \pi_{k} \leqslant \alpha
$$

is satisfied, where, as usual, we refer to some computable listing $\left\{\pi_{k}\right\}_{k \in \omega}$ of all $\Sigma_{a}^{-1}$-computable numberings. Without loss of generality, we may assume that $\pi_{0}(0)=\emptyset$. We write

$$
\pi_{k}^{s}(x)=\left\{y: f_{\pi_{k}(x)}(y, s)=1\right\} .
$$

We also assume that $\pi_{0}^{s}(0)=\emptyset$ for all $s$. As in the proof of Theorem 2.6, we use $\left\langle f_{\pi_{k}(x)}, \gamma_{\pi_{k}(x}\right\rangle$ to denote a $\Sigma_{a}^{-1}$-approximation (uniform in $k, x$ ) to $\pi_{k}(x)$. In the construction we will build an auxiliary sequence $\left\{g_{k}\right\}_{k \in \omega}$ of partial computable functions, each $g_{k}$ aiming at reducing $\pi_{k}$ to $\alpha$, if $\pi_{k}$ is a numbering of $\mathcal{A}$. Finally, let $a(k, x, i)$ be the values of some fixed computable injective function.

The construction. We begin with describing the strategy to meet $R_{k}$. The requirement will be spread into subrequirements $R_{k, x}$ : subrequirement $R_{k, x}$ aims at defining $g_{k}(x)$, if $\pi_{k} \in \operatorname{Comp}_{a}^{-1}(\mathcal{A})$. In defining $\mathcal{A}$, we will have care to achieve that if $\pi_{k} \in \operatorname{Comp}_{a}^{-1}(\mathcal{A})$ then there will be a unique $i$ such that $\pi_{k}(x)=\alpha(i)$, and we will let in this case $g_{k}(x)=i$.

Module. A reasonable module for satisfying $R_{k, x}$ is the following, carried out for each $i$ : 
1. let $a(k, x, i) \in \alpha(i)$;

2. await $a(k, x, i) \in \pi_{k}(x)$;

3. define $g_{k}(x)=i$ and extract $a(k, x, i)$ from all $\alpha(j), j \neq i$, if it lies in these sets;

4. await $a(k, x, i) \notin \pi_{k}(x)$ : if $a(k, x, i)$ gets extracted from $\pi_{k}(x)$;

5. enumerate $a(k, x, i)$ in all $\alpha(j)$, and go to $(2)$

Outcomes. Notice that we can not loop infinitely many times from (2) to (4), since $\pi_{k} \in \Sigma_{a}^{-1}$. Thus we distinguish the following outcomes:

1. for every $i$ we wait forever at (2), without ever passing through (4): then $\pi_{k}$ is not a numbering of $\mathcal{A}$, since for every $i, a(k, x, i) \in \alpha(i)-\pi_{k}(x)$;

2. there is some $i$ such that we move at some time from (2) to (4), and for this $i$ :

$\left(w_{1}\right)$ we wait forever at (2) (after being at (4)): then $a(k, x, i) \in \alpha(j)$, all $j$, but $a(k, x, i) \notin \pi_{k}(x)$, thus $\pi_{k}$ is not a numbering of $\mathcal{A}$;

$\left(w_{2}\right)$ we wait forever at $(4)$ : then $a(k, x, i) \in \alpha(i) \cap \pi_{k}(x)$, and $a(k, x, i) \notin$ $\alpha(j)$, if $j \neq i$; thus is $\pi_{k}$ is a numbering of the family, then $\pi_{k}(x)=$ $\alpha(i)$, consistently with our definition of $g_{k}(x)=i$, made at (3).

We give the detailed construction of $\alpha$ by stages: at stage $t$ we define $\alpha_{t}(e)$, or, by Definition 1.2 , the values $f(e, z, t)$ of a suitable computable function, together with the values $\gamma(e, z, t)$ of a partial computable mind-change function for $f: f$ and $\gamma$ will witness that $\alpha$ is a $\Sigma_{a}^{-1}$-computable numbering. At each stage, each parameter will retain the same value as at the preceding stage, unless otherwise explicitly redefined. Given $a, j$, at stage $s$ we say that we enumerate a in $\alpha(j)$, if we define $a \in \alpha_{s}(j)$; similarly, we say that we extract a from $\alpha(j)$, if we define $a \notin \alpha_{s}(j)$

Stage 0: Let $\alpha_{0}(e)=\emptyset$ and let $g_{k, 0}(e)$ be undefined for all $k$ and $e$. Moreover, let $f(e, z, 0)=0$, and $\gamma(e, z, 0) \uparrow$. Go to the next stage.

Stage $t+1$ : Let $m=(t)_{0}$ and suppose that $m=\langle k, x, i\rangle$. Carry out the instructions of Case 1 and Case 2, in the given order, and act accordingly: after acting go to next stage.

Case 1: If $t=\langle m, 0\rangle$ then enumerate the number $a(k, x, i)$ into $\alpha(i)$ : define $f(i, a(k, x, i), t+1)=1$ and $\gamma(z, t+1)=1$. (Recall that 1 is a notation of the ordinal 0.)

Case 2: If there exists $t^{\prime}<t$ such that $\left(t^{\prime}\right)_{0}=m$ then carry out one the following mutually exclusive subcases 2.1-2.3. 
Subcase 2.1: $a(k, x, i) \in \pi_{k}^{t+1}(x)$. Let $g_{k, t+1}(x)=i$ if $g_{k, t}(x)$ is undefined; for every $j \neq i$ extract $a(k, x, i)$ from $\alpha(j)$, defining $f(j, a(k, x, i), t+1)=0$ and

$$
\gamma(j, a(k, x, i), t+1)=\gamma_{\pi_{k}(x)}(a(k, x, i), t+1) .
$$

Subcase 2.2: $g_{k}^{t}(x)=i$ and $a(k, x, i) \notin \pi_{k}^{t+1}(x)$. For every $j \neq i$, enumerate $a(k, x, i)$ into $\alpha(j)$, define $f(j, a(k, x, i), t+1)=1$ and

$$
\gamma(j, a(k, x, i), t+1)=\gamma_{\pi_{k}(x)}(a(k, x, i), t+1) .
$$

Subcase 2.3: If Subcases 2.1, 2.2 do not hold then do nothing.

Verification. Notice that the only numbers that go into any of the sets numbered by $\alpha$ are numbers in the range of the function $a(k, x, i)$ : the element $a(k, x, i)$ is enumerated once for all in $\alpha(i)$, whereas its membership status in $\alpha(j)$, for $j \neq i$, is determined by the equation $\alpha(j)(a(k, x, i))=$ $\left.1-\pi_{k}(x)(a(k, x, i))\right)$ at all stages following the least stage $\langle\langle k, x, i\rangle, 0\rangle$ at which we attack $R_{k, x}$. The pair $\langle f, \gamma\rangle$ is a $\Sigma_{a}^{-1}$-computable approximation, since we redefine $\gamma(j, a(k, x, i))$ only after seeing that $f_{\pi_{k}(x)}(a(k, x, i))$ has changed, thus $\gamma_{\pi_{k}(x)}(a(k, x, i))$ has dropped, and in this case we let $\gamma(j, a(k, x, i))=$ $\gamma_{\pi_{k}(x)}(a(k, x, i))$. Moreover $a(0,0, i)$ is only contained in $\alpha(0)$. Finally, for every $k$, if $\pi_{k}$ is a numbering of $\mathcal{A}$, then $g_{k}$ is total and $\pi_{k}=\alpha \circ g_{k}$, since $\alpha\left(g_{k}(x)\right)$ is the only set of the family containing $a(k, x, i)$.

The above construction builds in fact an infinite family. As in [4] we can show that for every finite nonzero $n$ there is a family of $n$ sets: it suffices to build $\alpha(0), \ldots, \alpha(n-1)$ as above, and $\alpha(j)=\alpha(n-1)$, for every $j \geq n-1$.

\section{References}

[1] P. Alaev. To appear.

[2] M. M. Arslanov. Ershov hierarchy. Kazan, 2007. In Russian.

[3] C. J. Ash and J. Knight. Computable tructures and the Hyperarithmetical Hierarchy. Studies in Logic and the Foundations of Mathematics, 144, NorthHolland Publishing Co., Amsterdam, 2000.

[4] S. A. Badaev and Zh. T. Talasbaeva. Computable numberings in the hierarchy of Ershov. In Proceedings of 9th Asian Logic Conference, Novosibirsk, August 2005, S.Goncharov (Novosibirsk), H.Ono (Tokyo), and R.Downey (Wellington)(eds.). World Scientific Publishers. 2006, pp.17-30

[5] Yu. L. Ershov. A hierarchy of sets, I. Algebra and Logic, 7:47-73, 1968.

[6] Yu. L. Ershov. A hierarchy of sets, II. Algebra and Logic, 7:15-47, 1968. 
[7] Yu. L. Ershov. A hierarchy of sets, III. Algebra and Logic, 9:34-51, 1970.

[8] Yu. L. Ershov. Theory of Numberings. Nauka, Moscow, 1977. In Russian.

[9] S. Goncharov and A. Sorbi. Generalized computable numerations and nontrivial Rogers semilattices. Algebra and Logic, 36(6):359-369, 1997.

[10] S. Ospichev. Computable family of $\Sigma_{a}^{-1}$-sets without Friedberg numberings. In 6th Conference on Computability in Eueope, CiE 2010, 6th Conference on Computability in Europe, CiE 2010. Ponta Delgada, Azores, pages 311-315, 2010.

[11] T. Jech. Set Theory. Academic Press, New York, 1978.

[12] H. Rogers, Jr. Theory of Recursive Functions and Effective Computability. McGraw-Hill, New York, 1967. 